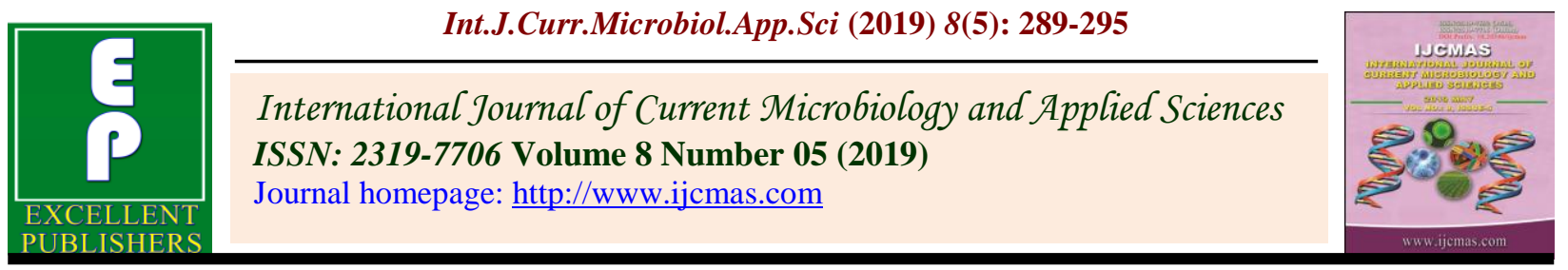

Original Research Article https://doi.org/10.20546/ijcmas.2019.805.034

\title{
Evaluation of Indian Mustard for their Potential Nutritional and Antinutritional Factors
}

\author{
Anubhuti Sharma* and P.K. Rai \\ ${ }^{I}$ ICAR-DRMR, Bharatpur, Rajasthan, India \\ *Corresponding author
}

\section{A B S T R A C T}

Keywords

Indian mustard, Nutritional and antinutritional factors,

Glucosinolates

Article Info

Accepted:

04 April 2019

Available Online:

10 May 2019
100 genotypes from germplasm were taken to evaluate oil content, total glucosinolate content, erucic acid, fibre content, phenol and sinigrin. The assayed genotypes contained 38.38- $42.89 \%$ oil, 38.97-113.01 $\mu$ mole/g glucosinolate, $18.67-47.05 \%$ erucic acid, 1.03-1.93\% Phenols, 7.82$14.58 \%$ fibre and sinigrin 9.40-107.34\% content using FT-NIR. The objective of this study is to characterize the large population of genotypes with advanced technique e.g. FT-NIR within short time period. Result clearly showed that seeds of core set of Indian mustard having high glucosinolates, grown at a field site revealed a wide variation in total concentrations of seed oil, erucic acid, phenols and mainly sinigrin.

\section{Introduction}

A large variability in nutritional quality parameters exists not only between different oilseed crops, but also within the same species. Nutritional quality of rapeseedmustard seed is determined by oil content and its fatty acid constituents and various antinutritional factors including glucosinolates, phytic acid, sinapine etc. These factors are also very important as it can be used to provide defense response in plants (Sharma et $a l ., 2016)$. Quality characteristics of rapeseedmustard oil have also been reported by earlier workers for various nutritional \& antinutritional factors (Anubhuti et al., 2017). These quality characteristics are important as these can further be used for breeding programs. However, breeders need large variability to initiate selection programs. Therefore, study of genetic diversity of nutritional \& antinutritional factors in brown mustard collection would help breeders in genotypic screening and selection in order to achieve high sinigrin level improvement. Sinigrin, a major aliphatic glucosinolate, is mainly responsible for acetylcholinesterase (AChE) inhibitory activities of Brassicaceae 
family (Ivica, 2014; Sharma et al., 2016). For this work few sensitive instruments can be used e.g. HPLC, GC-MS, NIR, FT-NIR etc. However Fourier transmission-Near Infrared Reflectance Spectroscopy is a rapid analytical technique results in many advantages, e.g. short time of analysis, low cost/sample ratios and no use of hazardous chemicals (Font et al., 2005).

However, till date major work has been done to decrease glucosinolates and erucic acid or to raise seed oil content. Other studies used molecular markers to structure genetic diversity for crop improvement programms. Nevertheless, these studies did not help breeders in the selection of genotypes to perform crosses to manage the glucosinolates/ sinigrin/erucic acid level in seed. However, few studies which focused on glucosinolates evaluation were done on local genotypes or performed on green tissues or both.

Therefore, this study aims to characterize biochemical traits e.g. nutritional \& antinutritional factors and their correlations in the large number of brassica genotypes.

\section{Materials and Methods}

\section{Plant material}

A core set of 100 genotypes of the brownseeded Indian mustard (Brassica juncea) with different traits for agronomic and nutritional characters were taken. Seeds of 100 genotypes were obtained from the germplasm section, ICAR-DRMR, Bharatpur.

In seeds, oil content, glucosinolates, erucic acid, fibre content and phenols have been estimated by Fourier transmission-Near Infrared Reflectance Spectroscopy (FT-NIR) (Bruker Optics, Ettlingen, Germany), a fast, reliable and rapid method which is generally used for screening the large number of genotypes. Data was rechecked in laboratory using spectrophotometric method. The dust free intact seeds (about $2 \mathrm{~g}$ ) of Brassica junce $a$ were packed in a standard ring cup and then scanned for oil content, glucosinolates, fibre content, phenols and erucic acid. The samples were scanned thrice to minimize the sampling error. However, sinigrin content was estimated directly by HPLC (Agilent 1100 series HPLC instrument with 6460 triple quad MS detector) through outsourcing (Directorate of Agricultural marketing, New Delhi).

The glass vials with seed samples were kept into sample holder for the spectral acquisition for FT-NIR measurement (Bruker Optics, Ettlingen, Germany) which is equipped with an integrative sphere, over the range 12,800$3600 \mathrm{~cm}^{-1}(780-2780 \mathrm{~nm})$ at $1 \mathrm{~nm}$ interval (Bala and Singh, 2013). Spectral acquisition was carried by OPUS spectroscopy software (v. 6.0 Bruker Optics, Ettlingen, Germany).

\section{Statistical analysis}

The statistical analyses were performed using Statistical Analysis System (SAS) JMP software version 9.0. The analysis of oil content, glucosinolate, erucic acid, phenol, fibre and sinigrin of each brassica genotype was based on three replications and the results are expressed as mean values \pm standard error (SE). For multi-factorial comparison, principal component analysis (PCA) and two way agglomerative hierarchical clustering (AHC) were used to display the correlations between the various parameters viz. oil content, glucosinolate, erucic acid, phenol, fibre and sinigrin of 100 brassica genotype.

\section{Results and Discussion}

Large genotypic variability was observed within the studied collection for all measured traits. The mean values for oil content were 
found to vary from the minimum of $38.38 \%$ in FA-77 to the maximum of $42.89 \%$ in FA63 with population mean of $41.01 \%$. As shown in Table 1 the higher oil content $(>42.00 \%)$ were found in grains of FA-4, FA-5, FA-12, FA-18, FA-25, FA-33, FA-49, FA-50, FA-53, FA-58, FA-63, FA-70, FA-71, FA-75, FA-88, FA-93 and FA-99 genotypes.

Lower phenol content $(<1.3 \%)$ were found in the grains of FA-8, FA-15, FA-59, FA-81 and FA-83 genotypes. The mean values for fiber content were found to vary from the minimum of $7.82 \%$ to $14.58 \mu \mathrm{g} / \mathrm{g}$. High fiber content $(>14 \%)$ were found in the grains of FA-52, FA-65, FA-94, FA-95 and FA-98 genotypes.

The mean values for total glucosinolate content were found to vary from the minimum of $38.97 \mu$ mole/g in inbred line FA-37 to the maximum of $113.01 \mu$ mole/g in FA-33 with population mean of $67.43 \mu$ mole/g. Lower glucosinolate content $(<40 \mu$ mole/g) were found in the grains of FA-37, FA-62 and FA75 genotypes. Large genotypic variability was observed within the studied collection for all measured traits (Table 1). The observed values for total glucosinolates were similar to those observed by Lionneton et al., (2004) within a mapping doubled haploid population. The concentration range of glucosinolates observed in our study was largely higher than those reported in other studies for glucosinolate content in mustard seeds (Ogbonnaya et al., 2003). However, most of those studies were focused on varieties devoted to oil and seedmeal production and with low glucosinolate contents (Bell et al., 2015; Beniwal et al., 2015). Wide variation in glucosinolate content among genotypes, also suggest differences in their health promoting properties and the opportunity for enhancement of their levels through genetic manipulation (Kushad et al., 1999).
The mean values for erucic acid content were found to vary from the minimum of $18.67 \%$ in inbred line FA-83 to the maximum of 47.05 $\%$ in FA-36 with population mean of $35.03 \%$. Lower erucic acid content $(<20 \%)$ were found in the grains of FA-10, FA-62, FA-75 and FA-83 genotypes. However revalidations of the results with other spectrophotometric methods are under progress.

The lower sinigrin content $(>15 \mu$ mole/g) were found in the grains of FA-37, FA-42, FA-45, FA-51 and FA-98 genotypes. However perusal of glucosinolate and sinigrin data clearly indicates the absence of a significant relationship between total glucosinolate and sinigrin content. This was also reported by Merah (2015). Detailed analysis of these glucosinolates showed large genotypic differences for both sinigrin and total glucosinolate levels in the collection. PCA analysis clearly depicts the relationship of sinigrin with total glucosinolates.

Principal Component Analysis (PCA) is a useful statistical technique to reveal the interrelationships between the different variables and to determine the optimum number of extracted principal components. The first principal component (PC1) had the highest Eigen value of 1.57 and accounted for 26.3 per cent of the total variation in the data set, while the second principal component (PC2) with Eigen value of 1.33 explained 22.1 per cent of the variation.

The projections of genotypes and traits are shown in PC1 and PC2 biplot (Fig. 1). In PCA, the length, direction and the angles between the lines indicate correlation between the variables or between variables and principal component axes (e.g., $\alpha=0^{0}$ and/or $180^{\circ}$ and $\mathrm{r}=1 ; \alpha=90^{\circ}$ and $\mathrm{r}=0$ ). The longer the line, the higher is the variance. The cosine of the angle between the lines approximates the correlation between the variables they 
represent. The closer the angle is to 90 or 270 degrees, the smaller the correlation. An angle of 0 or 180 degrees reflects a correlation of 1 or -1 , respectively (Fig. 1). All parameters occupied the right side of the biplot. Oil, erucic and phenol were observed on the right upper side of the biplot with high positive loading for both PC1 and PC2, while fibre, gls and sinigrin were grouped together on the right lower side of the biplot with positive loadings for PC1 and negative loadings for PC2. Significant positive correlation was observed between oil, erucic, phenol and sin. Sinigrin and gls also showed significant positive correlation $(r=0.449)$ with each other. Non significant negative correlation was observed among oil, sinigrin and phenol.

Table.1 Correlation between 100 genotypes of core set

$\begin{array}{lrrrrrr} & \text { oil } & \text { gls } & \text { erucic } & \text { phenol } & \text { fibre } & \text { SIN } \\ \text { oil } & 1.0000 & -0.0159 & 0.3550 & 0.1570 & -0.1196 & 0.0083 \\ \text { gls } & -0.0159 & 1.0000 & 0.1325 & -0.0190 & 0.0465 & 0.4487 \\ \text { erucic } & 0.3550 & 0.1325 & 1.0000 & 0.0116 & 0.1375 & 0.1419 \\ \text { phenol } & 0.1570 & -0.0190 & 0.0116 & 1.0000 & 0.0294 & 0.0030 \\ \text { fibre } & -0.1196 & 0.0465 & 0.1375 & 0.0294 & 1.0000 & 0.0691 \\ \text { SIN } & 0.0083 & 0.4487 & 0.1419 & 0.0030 & 0.0691 & 1.0000\end{array}$

Fig.1 Biplot analysis of 100 genotypes

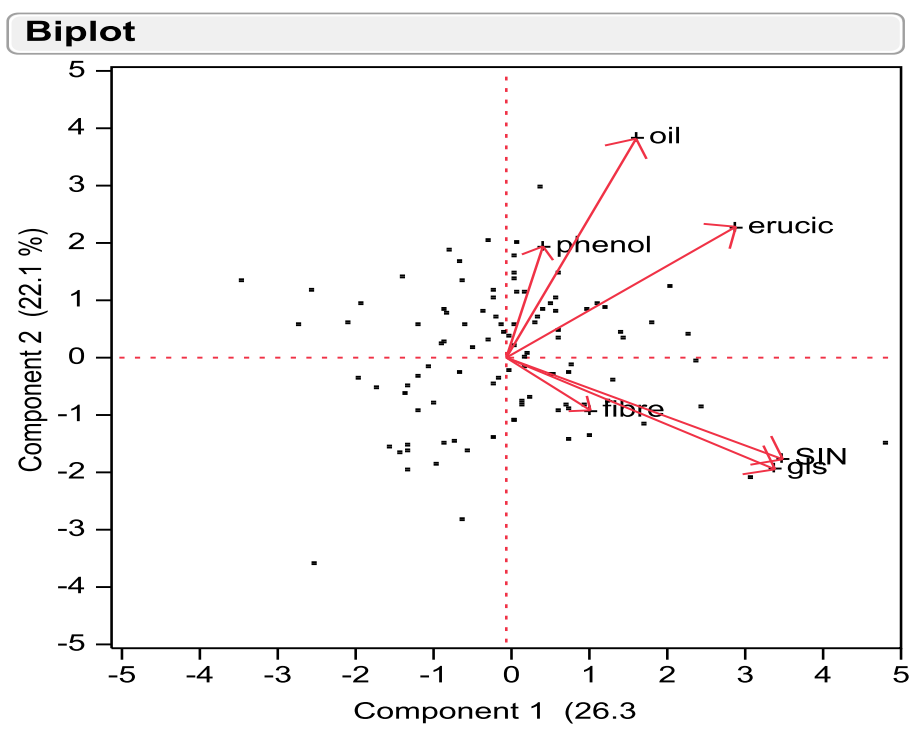

\section{Eigenvalues}

\begin{tabular}{|c|c|c|c|c|c|c|c|}
\hline Number & Eigenvalue & Percent & $\begin{array}{llll}20 & 40 & 60 & 80\end{array}$ & Cum Percent & ChiSquare & DF & Prob $>$ ChiSq \\
\hline 1 & 1.5791 & 26.318 & & 26.318 & 47.169 & 20.000 & 0.0006 * \\
\hline 2 & 1.3250 & 22.084 & & 48.403 & 31.466 & 14.000 & 0.0048 * \\
\hline 3 & 1.0366 & 17.277 & & 65.679 & 18.793 & 9.000 & 0.0270 * \\
\hline 4 & 0.9754 & 16.257 & & 81.937 & 12.068 & 5.000 & 0.0339 * \\
\hline 5 & 0.5541 & 9.234 & & 91.171 & 0.050 & 2.000 & 0.9754 \\
\hline 6 & 0.5297 & 8.829 & & 100.000 & 0.000 & 0.000 & \\
\hline
\end{tabular}


Fig.2 Dendrogram of the genotypes showing different groups of biochemical traits

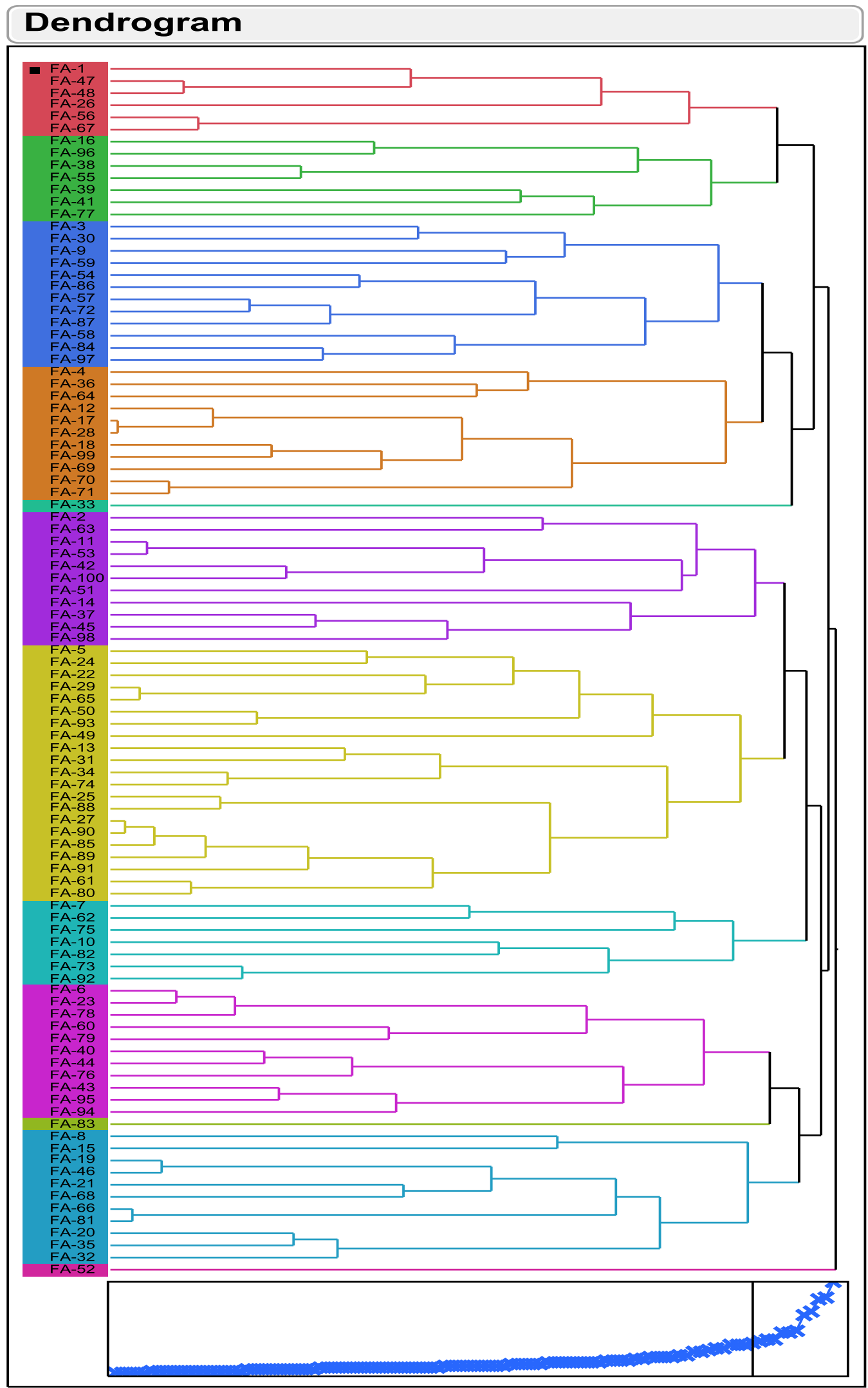


Three groups of hundred core set genotypes can be distinguished (Fig. 2). The first one is composed of 36 genotypes containing low sinigrin (less than $45 \mu$ mole/g of total GLS). Thirty nine genotypes composed the second group and are characterized by high level of sinigrin. Twenty four other genotypes constitute a third group with an intermediate level of sinigrin ranged from 35 to $65 \%$ of total GLS. Detailed analysis of these glucosinolates showed large genotypic differences for sinigrin levels in our collection. As previously reported sinigrin was consistently dominant over other glucosinolates (Rangkadilok et al., 2002; Merah, 2015). However few studies also showed that heavy seeds are poorer in sinigrin and tall plants are richer in sinigrin. These interesting correlations need further investigations to study the sinigrin level with vegetative growth in brassica.

In conclusion, the analytical analysis to characterize biochemical traits mainly aliphatic glucosinolate i.e. sinigrin with the help of FT-NIR and HPLC confirms the large genotypic variability for oil, glucosinolates, erucic acid and sinigrin within the seeds of Indian mustard collection from germplasm. These results are of interest for breeders so that they can combine both agronomic and nutritional traits to achieve high sinigrin content (approx 75-95 $\mu \mathrm{mol} . \mathrm{g}^{-1}$ ) and high seed yield.

In this study some genotypes (FA-3, FA-26, FA-33, FA-47, FA-49) showed high levels of sinigrin and could be used in breeding programs for improvement of glucosinolates mainly sinigrin level. Revalidations of the results are in progress.

\section{Acknowledgement}

The author is grateful to ICAR-DRMR, Bharatpur for financial \& technical help during experimentation.

\section{References}

Bell, L., Oruna-Concha, M. J., and Wagstaff, C. 2015. Identification and quantification of glucosinolate and flavonol compounds in rocket salad (Eruca sativa, Eruca vesicaria and Diplotaxis tenuifolia) by LC-MS: highlighting the potential for improving nutritional value of rocket crops. Food Chemistry. 172:852-861.

Beniwal, V., Aggarwal, H., Kumar, A., and Chhokar, V. 2015. Lipid content and fatty acid change in the developing silique wall of mustard (Brassica juncea L.), Biocatalysis and Agricultural Biotechnology. 4: 122125.

Font R, Del Rio-Celestino M, Cartea E, de Haro-Bailon A, (2005). Quantification of glucosinolates in leaves of leaf rape (Brassica napus ssp. pabularia) by near-infrared spectroscopy, Phytochemistry, 66: 175-185.

Ivica, B. 2014. Glucosinolates: Novel Sources and Biological Potential. Austin J Bioorg \& Org Chem. (1): 4.

Kushad, M.M., Brown, A.F., Kurilich, A.C., Juvik, J.A., Klein, B.P., et al., 1999. Variation of glucosinolates in vegetable crops of Brassica oleracea. J Agric Food Chem 47: 1541-1548.

Lionneton, E., Aubert, G., Ochatt, S., and Merah, O. 2004. Genetic analysis of agronomic and quality traits in mustard (Brassica juncea). Theoretical and Applied Genetics. 109 (4):792-799.

Manju Bala, Mhararaj Singh. 2013. Nondestructive estimation of total phenol and crude fibre content in intact seeds of rapeseed mustard using FTNIR. Industrial Crops and Products, 42: 357-362.

Merah, O. 2015. Genetic Variability in Glucosinolates in Seed of Brassica juncea: Interest in Mustard Condiment. 
Journal of Chemistry, Article ID 606142.

http://dx.doi.org/10.1155/2015/606142.

Ogbonnaya, F. C., Halloran, G., Pang, E., and

Gororo, N. 2003. Molecular analysis of genetic diversity in Brassica juncea and $B$. nigra germplasm accessions. In Proceedings of the 11th International Rapeseed Congress, pp. 489-491, Copenhagen, Denmark, July.

Rangkadilok, N., Nicolas, M. E., Bennett, R. N., Premier, R. R., Eagling, D. R., and Taylor, P. W. J. 2002. Developmental changes of sinigrin and glucoraphanin in three Brassica species (Brassica nigra, Brassica juncea and Brassica oleracea var. italica). Scientia Horticulturae. 96: 11-26.

Sharma, A., Kumar, A., Meena, H. S., and Singh, D. 2017. Chromatographic determination of phenolics in Brassica. Asian Journal of Chemistry. 29 (2): 296-300.

Sharma, A., Sharma, A.K., Yadav, P., and Singh, D. 2016. Isothiocyanates in Brassica: A potential anticancer agent. Asian pacific Journal of cancer prevention. 17 (9): 4507-4510.

\section{How to cite this article:}

Anubhuti Sharma and Rai, P.K. 2019. Evaluation of Indian Mustard for their Potential Nutritional and Antinutritional Factors. Int.J.Curr.Microbiol.App.Sci. 8(05): 289-295. doi: https://doi.org/10.20546/ijcmas.2019.805.034 